\title{
Das Buch Numeri und das Ende des Jahwisten Anfragen zur »Quellenscheidung« im vierten Buch des Pentateuch
}

\author{
Thomas Chr. Römer-Lausanne
}

\section{Martin Noth hatte Recht}

In Martin Noths Numerikommentar findet sich eine in letzter Zeit immer häufiger zitierte Feststellung: »Nimmt man das 4. Mosebuch für sich, so käme man nicht leicht auf den Gedanken an sdurchlaufende Quellen`, sondern eher auf den Gedanken an eine unsystematische Zusammenstellung von zahllosen Überlieferungsstücken sehr verschiedenen Inhalts, Alters und Charakters (>Fragmentenhypothese $\left(\right.$ ) ${ }^{1}$. Leider hat Noth aus dieser Einsicht nicht die nötigen Konsequenzen gezogen, sondern ist mit Ausnahme der Schlusskapitel von Num der Quellenscheidung treu geblieben. Diese herrschte ja zur Erscheinungszeit der ersten Auflage seines Numerikommentars in beinahe unangefochtener Souveränität über die Erforschung des Pentateuch. Die jüngsten Entwicklungen, die die seit einem Vierteljahrhundert währende Pentateuchdebatte nimmt, erweisen jedoch Noths Intuitionen als durchaus gerechtfertigt.

\section{Das Buch Numeri und die jüngste Pentateuchdebatte}

Die Diskussion um die Entstehung des Pentateuch ist seit einigen Jahren durch zwei Themen geprägt: zum einen ist die Frage nach Umfang, Autonomie und Intention der priesterlichen Grundschrift wieder völlig offen, zum anderen machen eine Reihe neuerer Veröffentlichungen die sogenannte »Fragmentenhypothese « wieder attraktiv. Diese neuen Trends sind nicht ohne Auswirkungen auf die Frage des »Jahwisten« und die der Entstehung des Numeribuches.

1 M. Noth, Das vierte Buch Mose. Numeri, ATD 7, ${ }^{3} 1977,8$. 


\subsection{Das Ende von ${ }^{g} "$}

Die klassische Hypothese, nach welcher das Ende der Priestergrundschrift in Dtn $34 \mathrm{zu}$ finden sei, ist von L. Perlitt mit m.E. überzeugenden Gründen als unhaltbar erwiesen worden ${ }^{2}$; daran kann auch die scharfsinnige Arbeit von C. Frevel nichts ändern ${ }^{3}$. In der Tat gibt es in Dtn 34 keine eindeutig priesterlichen Verse, Dtn 34,7-9 sind von bereits dtr beeinflussten spätpriesterlichen Texten abhängig. Die Einführung Josuas als Nachfolger Moses ist narratologisch gesprochen kaum einleuchtend, falls Dtn 34,7-9 als Ende der Priesterschrift konzipiert worden wären. Wenn nun aber das Schlusskapitel des Pentateuchs für letzteres nicht in Frage kommt, muss nach Alternativen gesucht werden. Eine Möglichkeit bestünde in einem P-Hexateuch Revival; d.h., man sucht in der Folge von Wellhausen das Ende von $\mathrm{P}^{\mathrm{g}}$ in Jos. Diese These ist in letzter Zeit hauptsächlich von N. Lohfink und E.A. Knauf vertreten worden, nach welchen dieser Abschluss in 18,1 bzw. 19,51 vorliege ${ }^{4}$, da in 18,1 eine Inklusion mit Gen 1,26-28 beabsichtigt werde. Dieses Argument ist nicht überzeugend, denn der Imperativ in Gen 1,28 richtet sich an die ganze Menschheit und hat den Erdkreis in Sicht, wohingegen es in Jos 18,1 um die Israeliten und ihr Land geht; dazu kommt, dass P einen theologischen (und literarischen) Unterschied zwischen der Ur- und der Volksgeschichte macht. Für die Geschichte Israels, die mit Abraham beginnt, steht der priesterliche Programmtext in Gen 17; Jos 18,1 oder 19,51 bieten jedoch keinerlei Anspielung auf diesen Text ${ }^{5}$. Weiterhin ist zu bemerken, dass eine abschließende Niederlassung der Israeliten in Silo aus der Perspektive von $P$ keinen rechten Sinn macht. Die sogenannten P-Stellen im Buch Josua sind eher als dtr-priesterliche Mischtexte zu bezeichnen, deren stilistische Propria nicht

2 L. Perlitt, Priesterschrift im Deuteronomium? (1988), in: Deuteronomium-Studien, FAT 8, 1994, 123-143.

3 C. Frevel, Mit Blick auf das Land die Schöpfung erinnern. Zum Ende der Priestergrundschrift, HBS 23, 1999; ders., Ein vielsagender Abschied. Exegetische Blicke auf den Tod des Mose in Dtn 34,1-12, BZ 45 (2001), 209-234. Die Malaise wird deutlich bei einem weiteren Befürworter von Dtn 34, der von einem »höchst eigenwilligen Schluss« spricht ( $P$. Weimar, Art. Priesterschrift, NBL 11, 1997, Sp. 168-171,170).

4 Für 18,1: E.A. Knauf, Die Priesterschrift und die Geschichten der Deuteronomisten, in: T. Römer (Hg.), The Future of the Deuteronomistic History, BEThL 147, 2000, 101-118, vgl. auch H. Seebass, Art. Pentateuch, TRE 26, 1996, 185-209, 192; für 19,1: N. Lohfink, Die Priesterschrift und die Geschichte (1977) in: ders., Studien zum Pentateuch, SBAB 4, 1988, 213-253; vgl. auch J. Blenkinsopp, The Pentateuch. An Introduction to the First Books of the Bible, 1992, 237.

5 So zu Recht, J.L. Ska, Introduction à la lecture du Pentateuque, Le livre et le rouleau 5 , $2000,214$. 
mit $\mathrm{P}^{\mathrm{g}}$ konform gehen. Wenn das Ende von $\mathrm{P}^{\mathrm{g}}$ weder in Dtn noch in Jos vorliegt, muss eine reduzierte Priestergrundschrift ins Auge gefasst werden. Bisweilen wird ein Abschluss von $\mathrm{P}^{\mathrm{g}}$ in Num vorgeschlagen, und zwar meistens in 27,12236. Aber diese Perikope ist gleichermassen unabgeschlossen wie Dtn 34,7-9 und kommt deshalb ebenso wenig in Frage. Auch andere Kandidaten in Num, wie z.B. Num 20, Num 14 oder das Ende der Sinaiperikope in Num 10,11f stellen keine überzeugende Endpunkte dar ${ }^{7}$. Damit muss der Abschluss von $\mathrm{P}^{\mathrm{B}}$ in der Sinaiperikope gesucht werden ${ }^{8}$. Es mehren sich in der Tat die Stimmen, die Priestergrundschrift in Ex 40 enden zu lassen ${ }^{9}$. Die Errichtung eines Heiligtums für Jhwh stellt religionsgeschichtlich gesehen ein passendes Finale dar. Dieses wird dadurch noch unterstrichen, dass, wie bereits des öfteren bemerkt wurde, Ex 2540 eine Reihe von Motivaufnahmen aus Gen 1 beinhalten ${ }^{10}$. Es bleibt allerdings $\mathrm{zu}$ fragen, ob ein Ende von $\mathrm{P}^{\mathrm{g}}$ ohne Einsetzung der Priesterdynastie denkbar ist. Insofern wäre als Abschluss der Priestergrundschrift Lev 9 zu erwägen ${ }^{11}$. Lev 9,24 ist in der Tat ein gut vorstellbares Finale, und die dort erwähnte Proskynese stellt möglicherweise einen Rückverweis auf Abrahams Reaktion in Gen 17 dar,

6 So zuletzt Ska, ebd., 215.

7 Vgl. die einleuchtenden Ausführungen von Frevel, Blick (s. Anm. 3), 75-82.

8 Dies umso mehr als die priesterlichen Texte in Numeri gewisse von $P^{g}$ abweichende Charakteristika aufweisen; vgl. dazu zuletzt T. Pola, Die ursprüngliche Priesterschrift. Beobachtungen zur Literarkritik und Traditionsgeschichte von $\mathrm{P}^{\mathrm{g}}$, WMANT 70, 1995, 56-92. Die Darstellung Israels als ecclesia militans in Num 1-10 ist schlecht mit dem oft gerühmten Pazifismus von $\mathrm{P}^{\mathrm{g}}$ in Einklang zu bringen, und die Rebellionsgeschichten in Num 13-14; 16-17 und 20 stehen in einem gewissen Widerspruch zur Anthropo- und Theologie von $\mathrm{P}^{\mathrm{g}}$ wie sie im Exodusbuch zum Ausdruck kommt. Dort gibt es für den Menschen keine Möglichkeit gegen Gottes Projekte Widerstand zu leisten (vgl. auch die Überlegungen bei E. Aurelius, Der Fürbitter Israels. Eine Studie zum Mosebild im Alten Testament, CB.OT 27, 1988, 187-202 und O. Kaiser, Der Gott des Alten Testaments. Theologie des Alten Testaments I, UTB 1747, 1993, 180-182).

9 Insbesondere Pola, Priesterschrift (Ex 40,16-17a.33b), ähnlich Michaela Bauks, La signification de l'espace et du temps dans »l'historiographie sacerdotale«, in: The Future (s. Anm. 4), 29-45; R.G. Kratz, Die Komposition der erzählenden Bücher des Alten Testaments, 108 (Ex 40,34). Eine noch radikalere Lösung schlägt E. Otto, Forschungen zur Priesterschrift, ThR 62 (1997), 1-50; 25-27, 36, vor. Er sieht das Ende von $\mathrm{P}^{\mathbb{g}}$ in Ex 29,42b-46; damit fällt jedoch die Inklusion mit Gen 1 weg.

10 Vgl. bereits M. Buber, Der Mensch von heute und die jüdische Bibel, in: M. Buber - F. Rosenzweig, Die Schrift und ihre Verdeutschung, 1936, 13-45.39-41, sowie die Zusammenstellung der Parallelen bei J. Blenkinsopp, Pentateuch (s. Anm. 4), 218.

11 So E. Zenger, Einleitung in das Altes Testament, Studienbücher Theologie 1,1, 1995, 95; ders., Art. Priesterschrift, TRE 27, 1997, 435-446; 438f. 
wo Gott sich dem Stammvater Israels offenbart (Gen 17,3)12. Die Entscheidung zwischen Ex 40 oder Lev 9 kann im Rahmen des hier zu verhandelnden Problems offen bleiben. Im einen oder im anderen Fall wird nämlich die Idee, dass die Priestergrundschrift das Skelett für den ganzen Pentateuch darstellt ${ }^{13}$, hinfällig.

\section{2. "Jahwistisches Geschichtswerk» oder Fragmentenhypothese}

So bleibt als möglicher Kandidat für das narrative Gerüst des Pentateuchs der Jahwist bzw. der zum Deuteronomisten gemauserte Jahwist. Diese Idee hatte bereits, allerdings im Rahmen des Hexateuchs, G. von Rad vertreten, der im Jahwisten denjenigen sah, der »dem ganzen Hexateuch seine Gestalt und sein Maß gab « ${ }^{14}$. Von Rad hat einen würdigen Nachfolger in J. Van Seters gefunden, selbst wenn dieser den Jahwisten einige Jahrhunderte später ansetzt ${ }^{15}$. Die Verteidiger des Jahwisten, sei er nun vorexilisch (H. Seebass, W.H. Schmidt) ${ }^{16}$, exilisch (C. Levin, Van Seters) ${ }^{17}$ oder früh-nachexilisch (M. Rose) ${ }^{18}$ teilen im Grunde dasselbe Postulat. Ähnliches gilt auch für das »Kompositionsmodell«, welches den Pentateuch als Kombination einer D- und einer P-Komposition ansieht, vor allem, wenn die D-Komposition als ein $\mathrm{P}$ vorausgehendes Werk verstanden wird (Blum, Carr) ${ }^{19}$. Allen diesen Ansätzen ist gemein, dass ein von Gen bis mindestens nach Num reichender vorpriesterlicher Erzählzusammenhang

12 Das Thema des Segens in V. 23 kann ebenfalls als ein über Ex 40 laufender Rückbezug auf Gen 1 verstanden werden, auch der achte Tag in V. 1 erklärt sich gut in demselben Rahmen.

13 M. Noth, Überlieferungsgeschichte des Pentateuch, 1948, 7-9; A. de Pury, Abraham: The Priestly Writer's »Ecumenical« Ancestor, in: S.L. McKenzie/T. Römer (Hg.), Rethinking the Foundations. Historiography in the Ancient World and in the Bible, FS J. Van Seters, BZAW 294, 2000, 163-181.

14 Genesis, ATD 2/4;1972, $3-4$; grundlegend: Das formgeschichtliche Problem des Hexateuch (1938), 1971.

15 Vgl. J. Van Seters, Prologue to History. The Yahwist as Historian in Genesis, 1992; The Life of Moses. The Yahwist as Historian in Exodus-Numbers, 1994. Interessant ist auch folgende Bemerkung G. von Rads: "man mag den Jahwisten zeitlich ansetzen wann man will; gemessen an dem Alter der von ihm verarbeiteten Stoffe bedeutet er eine späte Phase« (Genesis, 5).

16 Seebass, Pentateuch (s. Anm. 4); W.H. Schmidt, Exodus 7,1-10,29, BK.AT II.2, 1995.

17 C. Levin, Der Jahwist, FRLANT 157, 1993; Van Seters (s. Anm. 15).

18 M. Rose, Deuteronomist und Jahwist: Untersuchungen zu den Berührungspunkten beider Literaturwerke, AThANT 67, 1981.

19 E. Blum, Studien zur Komposition des Pentateuch, BZAW 189, 1990; D.M. Carr, Reading the Fractures of Genesis, 1996. 
angenommen wird ${ }^{20}$. Schaut man jedoch genauer hin, zeigt sich schnell, dass dieser Zusammenhang sehr unterschiedlich charakterisiert wird. Für die einen handelt es sich um »deuteronomistischen« Stil und Theologie, für die anderen haben die vorpriesterlichen Texte des Tetrateuchs mit der dtr Sprach- und Gedankenwelt sehr wenig zu tun. Vielleicht ist diese nicht unerhebliche Divergenz ein Indikator für die Schwierigkeit ein Gen, Ex und Num umfassendes vorpriesterliches Dokument zu postulieren ${ }^{21}$.

Diese Schwierigkeit wird durch neuere Veröffentlichungen bestätigt, welche erneut eine Fragmentenhypothese in Erwägung ziehen. Die schon von F. Crüsemann $^{22}$ unterstrichene Selbstständigkeit der vorpriesterlichen Urgeschichte ist durch die Untersuchung von $\mathrm{M}$. Witte ${ }^{23}$ bestätigt worden, der aufgezeigt hat, dass die »jahwistische« Urgeschichte (welche er frühnachexilisch datiert) erst durch $\mathrm{P}$ mit den anderen Pentateuchtraditionen verbunden wurde ${ }^{24}$; diese These wird dadurch erhärtet, dass die Verbindungsverse in Gen 12,1-3 nach der jüng-

20 Eine bemerkenswerte Abweichung von der gängigen Nomenklatur findet sich bei Kratz, Komposition (s. Anm. 9), passim. »J « liegt bei ihm nur in den vorpriesterlichen Texten der Genesis vor, wohingegen jene in Ex bis Jos als »E« bezeichnet werden. Hier ist zu Recht dem Einschnitt zwischen Gen und Ex Rechnung getragen, s.u.

21 Wenn man von der Gen ausgeht, ist der sog. Jahwist recht wenig dtr, dafür umso mehr in Ex.

22 F. Crüsemann, Die Eigenständigkeit der Urgeschichte. Ein Beitrag zur Diskussion um den "Jahwisten «, in: J. Jeremias/ L. Perlitt (Hg), Die Botschaft und die Boten, FS H.W. Wolff, 1981, 11-29.

23 M. Witte, Die biblische Urgeschichte. Redaktions- und theologiegeschichtliche Beobachtungen zu Genesis 1,1-11,26, BZAW 265, 1998.

24 Mehrere Exegeten erwägen jetzt einen nachpriesterlichen Ursprung der sog. 》J $₫(-T e x t e$ der Urgeschichte, welche sich am einfachsten als eine Redaktion der P-Erzählung verstehen liessen (so v.a. J. Blenkinsopp, Pentateuch [Anm. 4], 54ff; J.L. Ska, El Relato del diluvio. Un relato sacerdotal y algunos fragmentos redaccionales posteriores, EstB 52 (1994) 37-62; E. Otto, Die Paradieserzählung Genesis 2-3: Eine nachpriesterliche Lehrerzählung in ihrem religionshistorischen Kontext, in: A.A. Diesel u. a. (Hg.), 》Jedes Ding hat seine Zeit...«. Studien zur israelitischen und altorientalischen Weisheit, FS D. Michel, BZAW 241, 1996, 167-192; T. Krüger, Das menschliche Herz und die Weisung Gottes. Elemente einer Diskussion über die Möglichkeiten und Grenzen der Tora-Rezeption im Alten Testament, in: T. Krüger/R.G. Kratz (Hg.), Rezeption und Auslegung im Alten Testament und seinem Umfeld, OBO 153, 1997, 65-92). Einige der sog. " J «-Texte in Gen 1-11 verstehen sich in der Tat besser als redaktionelle Ergänzungen, dies ist $\mathrm{m}$.E. jedoch nicht der Fall für alle nichtpriesterlichen Verse in der Urgeschichte, aus denen sich, wie Witte gezeigt hat, durchaus ein autonomer Zyklus rekonstruieren lässt. 
sten Ananlyse von J.L. Ska ${ }^{25}$ sehr wahrscheinlich als ein nachpriesterlicher theologischer Deutungstext zu verstehen sind.

Für die Auszugserzählung in Ex 1-15 hat man ebenfalls seit längerer Zeit eine ursprüngliche Autonomie postuliert ${ }^{26}$. Diese Idee ist durch die Arbeit von J.C. Gertz untermauert worden. Ihm zufolge sind in Ex 1-15 »die expliziten Rückbezüge auf die Genesis sämtlich als priesterschriftlich oder nachpriesterschriftlich« zu bezeichnen ${ }^{27}$. Dies würde bedeuten, dass die literarische Verbindung zwischen Gen und Ex erstmalig von $\mathrm{P}$ vorgenommen wurde. Diese These hat nun Konrad Schmid in einer umfangreichen Untersuchung verteidigt. Erst durch Texte wie Gen 17; Ex 2,23-25 und Ex 6,2-8 würde unverkennbar eine literarische Verbindungslinie zwischen der Patriarchen- und der Exodusüberlieferung gezogen $^{28}$. Wenn sich eine solche Sicht bewähren sollte, wäre ein radikales Umdenken in Bezug auf die herkömmlichen Pentateuchmodelle notwendig. Es müsste insbesondere überlegt werden, ob nicht im Hinblick auf mögliche Entstehungsmodelle viel mehr zwischen den einzelnen Textkomplexen bzw. Büchern differenziert werden sollte, als dies bisher geschieht.

\subsection{Numeri als Bindeglied zwischen "priesterlichem « Tetrateuch und Deutero- nomistischen Geschichtswerk}

Die Frage woher die Einteilung der Torah in fünf Bücher kommt, ist noch nicht geklärt ${ }^{29}$. Die Rollenfunde in Qumran setzen bereits eine solche voraus. Gegenüber der herkömmlichen Sicht wäre zu überlegen, ob die Unterscheidung von fünf Rollen nicht zumindest teilweise der Publikation des Pentateuch vorausgeht. Wenn die Ur-, Väter- und Exodusgeschichten relativ lange in autonomer Weise überliefert wurden, kann man sich ihre (vorpriesterliche ?) Zusammenstellung in separaten Rollen durchaus vorstellen. Der eigenständige Charakter der einzelnen

25 J.L. Ska, L'appel d'Abraham et l'acte de la naissance d'Israël. Genèse 12,1-4a, in: M. Vervenne/J. Lust (Hg.), Deuteronomy and Deuteronomic Literature, FS C.H.W. Brekelman, BEThL 133, 1997, 367-389.

26 Vgl. bereits J. Pedersen, Passahfest und Passahlegende, ZAW 52 (1934), 161-175; weiter E.A. Knauf, Midian. Untersuchungen zur Geschichte Palästinas und Nordarabiens am Ende des 2. Jahrtausends v.Chr., ADPV, 1988, 131-135; R. Albertz, Religionsgeschichte Israels in alttestamentlicher Zeit, GAT 8, 1992, 71-72.

27 J.C. Gertz, Tradition und Redaktion in der Exoduserzählung. Untersuchungen zur Endredaktion des Pentateuch, FRLANT 186, 1999, 382.

28 K. Schmid, Erzväter und Exodus. Untersuchungen zur doppelten Begründung der Ursprünge Israels innerhalb der Geschichtsbücher des Alten Testaments, WMANT 81, 1999, 67-75.

29 Vgl. zuletzt die Überlegungen bei Schmid, ebd., 26-33. 
Bücher ist in der Tat strukturell und inhaltlich leicht zu erkennen. Für die Genesis ist zunächst zu bemerken, dass die wohl priesterliche Toledotstruktur im Grunde auf dieses Buch beschränkt ist ${ }^{30}$. Dies stellt ein Indiz für die Anerkennung der Eigenständigkeit des Buches dar, noch zu der Zeit als jenes bereits den ersten Teil des priesterlichen Werkes bildete. Auch das Buch Exodus stellt eine klar erkennbare Einheit dar. Es führt »de la servitude au service ${ }^{31}$ und stellt gewissermassen die Geschichte eines Herrschaftswechsels dar. Israel, zu Anfang des Buches ' $c b c e d$ Pharaos, wird am Ende zum 'cebced Jhwhs. Der Konstruktion von Bauten für Pharaon in Ex 1 entspricht als Gegenprojekt die Konstruktion des Heiligtums für Jhwh in Ex 35-40. Levitikus hebt sich durch das Fehlen fast jeglicher Narration und die Zusammenstellung verschiedener Sammlungen von überwiegend priesterlichen Vorschriften von den anderen Büchern der Torah ab. Auch das Deuteronomium ist als Moserede klar als eine eigenständige Einheit zu verstehen. Das einzige Buch, dessen Struktur und Kohärenz nicht sogleich klar werden, ist somit Numeri. Dies zeigt zunächst die im Unterschied zu den anderen Büchern geführte Debatte um die Unterteilung der vierten Torahrolle, für welche keinerlei Konsens in Sicht ist. Ist Numeri wegen der zweifachen Volkszählung zweizuteilen (Num 1-25; 26-36) ${ }^{32}$, oder ist mit der Mehrheit der Forscher aus geographischen Gründen eine Dreiteilung vorzuziehen, für deren $\mathrm{Ab}-$ grenzungen jedoch wiederum unterschiedliche Vorschläge gemacht werden ${ }^{33}$ ? Auch inhaltlich ist Num weit weniger kohärent als Gen, Ex, Lev oder Dtn ${ }^{34}$. Man kann relativ leicht mittelgrosse Einheiten wie den Rebellionszyklus in Num 11-

30 Die einzige Ausnahme im Pentateuch stellt Num 3,1 dar (Toledot Moses und Aarons). Dieser Text ist als eine späte Imitation der Genesisüberschriften zu verstehen.

31 G. Auzou, De la servitude au service. Etude du livre de l'Exode, Connaissance de la Bible, 1961.

32 So insbesondere D.T. Olson, The Death of the Old and the Birth of the New. The Framework of the Book of Numbers and the Pentateuch, Brown Judaic Studies 71, 1985.

33 Vgl. die Diskussion bei O. Artus, Etudes sur le livre des Nombres. Récit, Histoire et Loi en Nombres 13,1-20,13, OBO 157, 1997, 15-18 und H. Seebass, Numeri, BK.AT IV, 1993ff., 6.

34 Anders Seebass, Numeri, 148-152; ders., Die Ankündigung des Mosetodes (noch unveröffentlicht. Ich danke Herrn Kollegen Seebass furr die freundlicherweise gewährte Einsicht in das Manuskript). Seebass beruft sich auch auf Mary Douglas, In the Wilderness. The Doctrine of Defilement in the Book of Numbers, JSOT.S 158, 1993. Die von ihr aufgezeigten "patterns « (bes. 102-126) sind eher als ein Meta-Schema zu verstehen und nicht als ein klar erkennbares Strukturprinzip. Allerdings ist Douglas dahingehend recht zu geben, dass Numeri als ein »Kommentar« zu Genesis (und anderen Pentateuchbüchern) verstanden werden kann (98-101). 
20 oder die Bileamsgeschichte in 22-24 unterscheiden; ein übergreifendes Thema ist jedoch schwer auszumachen.

Diese Sonderstellung von Num kann zusammen mit den vorgetragenen Beobachtungen zum Ende von $\mathrm{P}^{\mathrm{g}}$ und zur teilweisen Rückkehr der Fragmentenhypothese zu folgender Überlegung ausgebaut werden: Der erste »Pentateuch« war ein Tritoteuch, d.h. die Zusammenstellung der Traditionen in Gen-Lev ${ }^{35}$ unter priesterlicher Federführung. Unabhängig davon gab es ein von Dtn bis Kön reichendes »Deuteronomistisches Geschichtswerk « ${ }^{36}$. Im Rahmen der Bemühungen um ein breit akzeptierbares Gründungsdokument des in der Perserzeit entstehenden Judentums kam es zu einem Kompromiss zwischen priesterlichen und deuteronomistisch-laizistischen Kreisen ${ }^{37}$. Dabei steuerten die Deuteronomisten hauptsächlich das Deuteronomium und deren priesterliche Kollegen ihren von der Gründung der Welt bis zur Gründung des Heiligtums reichenden Bericht (Gen-Lev) bei. Bei dieser Zusammenfügung wurde das Buch Numeri zu einer Art Brücke zwischen Tetrateuch und dem vom DtrG abgetrennten Dtn ${ }^{38}$. Das

35 Man kann ein ursprüngliches Ende von $P^{\mathrm{g}}$ z.B. in Lev 9 annehmen und die folgenden Kapitel des Buches als eine innerpriesterliche Weiterschreibung erklären (Kratz, Komposition [s. Anm. 9], 116: »Nachträge im Rahmen der noch selbständigen Priesterschrift«).

36 Im Gegensatz zum Jahwisten ist die Idee eines DtrG trotz einiger Grabredner noch nicht gestorben, selbst wenn Noths Auffassung desselben erheblich zu korrigieren ist, vgl. dazu T. Römer, La fin de l'historiographie deutéronomiste et le retour de l'Hexateuque?, ThZ 57 (2001), 269-280.

37 Die Kompromissidee ist heutzutage weit verbreitet. Allerdings gehen die Ansichten über die Bestimmung dieses Kompromisses ebenso weit auseinander, vgl. z.B. Blum, Studien (s. Anm. 19), passim; Albertz, Religionsgeschichte (s. Anm. 26); E.A. Knauf, Audiatur et altera pars. Zur Logik der Pentateuchredaktion, BiKi 53 (1998), 118-126. Auf die sogenannte Reichsautorisation braucht hier nicht näher eingegangen zu werden. Selbst wenn diese These aufgegeben bzw. erheblich modifiziert werden müsste (vgl. dazu die Diskussion zwischen P. Frei, J. Blenkinsopp, L.L. Grabbe, G.N. Knoppers, J.L. Ska in: J.Watts [Hg.], Persiah and Torah, The Theory of the Imperial Autorisation of the Pentateuch, SBL.SS 17, 2001; und weiter U. Rüterswörden, Die persische Reichsautorisation der Thora: Fact or Fiction ?, ZAR 1 [1995], 47-61), würde dadurch die Interpretation des Pentateuch als eines Kompromisses verschiedener ideologischer Gruppen nicht hinfällig (vgl. dazu wegweisend bereits B.J. Diebner, Zur Funktion der kanonischen Textsammlung im Judentum der vorchristlichen Zeit. Gedanken zu einer Kanon-Hermeneutik, DBAT 22 [1985], 58-73).

38 Die Abtrennung des Dtn von den folgenden Büchern erklärt sich dadurch, dass die Gründungsurkunde des Judentums letztendlich als Torah, d.h. als die (allein) von Mose vermittelte Unterweisung verstanden wurde, im Gegensatz zu dem alternativen Entwurf eines Hexateuchs, in welchem das Land als entscheidende Heilsgabe in den Mittelpunkt rückt, vgl. dazu T. Römer/M.Z. Brettler, Deuteronomy 34 and the Case For a Persian Hexateuch, JBL 119 (2000), 401-419 und E. Otto, Das Deuteronomium im Pentateuch und Hexateuch, FAT $30,2000,243-246$. 
bedeutet, dass Numeri als das letzte Buch der Torah entstanden ist. Diese Hypothese ermöglicht die Erklärung folgender Befunde:

Numeri weist unbestreitbar weit mehr dtr-priesterliche Mischtexte auf, als die anderen Bücher des Pentateuchs, in welchen eine literarkritische Aufteilung in verschiedene Schichten kaum mehr zu erreichen ist, wie z.B. Num 16-17; 25 und 32. Sind solche Texte von vornherein so konzipiert, um »dtr« und »priesterlichen« Anliegen zugleich Rechnung zu tragen? Weiterhin finden sich in Num Ergänzungen bzw. Aktualisationen, die eigentlich besser an anderen Stellen des Pentateuchs Platz hätten, wie z.B. die Passahbestimmungen in Num 9, die man sinnvoller nach oder in Ex 12 oder Lev 23 hätte integrieren können oder die Nachträge zur Einweihung des transportablen Heiligtums in Num 7, die eigentlich nach Ex 40 (vgl. Num 7,1) ihren Platz gehabt hätten. Anscheinend konnten diese Texte nur noch im Numeribuch Aufnahme finden, da die anderen vier Bücher wohl schon einen protokanonischen Status innehatten. In der Tat schliessen Lev 26,46 bzw. 27,34 die Sinaioffenbarung eindeutig ab: »Das sind die Gebote, die Jhwh dem Mose auf dem Berg Sinai für die Israeliten gab«. Man hat also im Nachhinein die Sinaiperikope durch Num 1-10 ausgeweitet und diese Verlängerung mit einer neuen Überschrift versehen, welche anscheinend eine Differenz zu den in Ex-Lev gegebenen Gesetzen impliziert: »Und Jhwh redete mit Mose in der Wüste Sinai im Zelt der Begegnung«. Die auf Num 1,1 folgende Jhwhworte werden im Gegensatz zu Ex-Lev Mose nicht mehr auf dem Berg, sondern in der Wüste im transportablen Zelt übermittelt; dadurch wird ein qualitativer und diachroner Unterschied zum Vorangehenden markiert ${ }^{39}$. So wird die These der Existenz eines protokanonischen Tetrateuchs weiter abgestützt.

Die Eigenständigkeit eines Tetrateuchs ist vielleicht auch bei Hekataios von Abdera belegt. Dessen bei Diodoros überlieferte Geschichte Moses enthält die Feststellung, dass Moses den Israeliten ein Gesetz gegeben hat »an dessen Ende folgende Worte geschrieben stehen: `Dies sind die Worte die Moses von Gott gehört und den Israeliten übermittelt hat « « ${ }^{40}$. T. Reinach interpretiert dies als eine freie Wiedergabe von Lev 27,3441, was bedeuten würde, dass noch am Ende des 4. Jh. v. Chr. die Erinnerung an einen ehemals selbständigen Tetrateuch bestand. Es sei ebenfalls

39 Vgl. dazu die Ausführungen von D.T. Olson, Death (s. Anm. 32), 1985, 48-49.

40 F. Jacoby, Die Fragmente der Griechischen Historiker, III/A, 1940, 14.

41 T. Reinach, Textes d'auteurs grecs et romains relatifs au judaïsme, 1895, 18, Anm. 2. Vgl. auch L.L. Grabbe, Jewish Historiography and Scripture in the Hellenistic Period, in: L.L. Grabbe (Hg.), Did Moses Speak Attic? Jewish Historiography and Scripture in the Hellenistic Period, JSOT.S 317, 2001, 129-155. 
erwähnt, dass anscheinend Num später als Gen, Ex und Lev ins Griechische übersetzt wurde ${ }^{42}$ und dass Num das in Qumran am wenigsten bezeugte Buch des Pentateuch ist ${ }^{43}$. Diese Beobachtungen dürfen natürlich nicht überinterpretiert werden. Im Zusammenhang mit den obigen Erwägungen stellen sie jedoch ein zusätzliches Argument für eine autonome Entstehung von Gn-Lev* dar.

Wenn nun das Buch Num als späte Brücke zwischen Tetrateuch und Dtn entstand, wird man die nicht-priesterlichen Texte dieses Buches kaum einem Jahwisten zuschreiben können.

\section{3. »Fortschreibung« statt »Jahwist« in Numeri}

Martin Noth hatte die letzen Kapitel des Numeribuches (27-36) als nachpriesterliche Zufügungen unterschiedlicher Herkunft betrachtet und dabei bemerkt: »man wird damit rechnen müssen, daß die einzelnen Stücke einfach so aneinandergereiht wurden, wie sie eines nach dem anderen hinzukamen « ${ }^{44}$. Damit hatte er bereits im Grunde genommen ein Fortschreibungsmodell für das Ende von Num ins Auge gefasst. Ein solches Model kann m.E. auf die Entstehung des ganzen Buches angewendet werden. Dies soll kurz in bezug auf die ersten nichtpriesterlichen Texte von Num dargestellt werden.

\subsection{Das Problem des Jahwisten in Num 11-12}

Neuere mit der Hypothese des Jahwisten arbeitenden Untersuchungen zu Num 11-12 sind sich in der Bestimmung von $\mathbf{J}$ in diesen Kapiteln keineswegs einig. H. Seebass folgt der traditionnellen Unterscheidung in Ältesten- und Wachtelerzählung, und will, wenn ich ihn recht verstehe, beide Textstränge als ursprünglich selbständige Erzählungen verstehen und sie beide $\mathrm{J}$ zuschreiben, wobei die Geschichte von der Wachtelsendung ursprünglich hinter Num 16* $(\mathrm{J})$ gestanden

42 Vgl. M. Rösel, Die Septuaginta und der Kult. Interpretationen und Aktualisierungen im Buch Numeri, in: Y. Goldman/C. Uehlinger (Hg.), La double transmission du texte biblique, FS A. Schenker, OBO 179, 25-40, 28.

43 Gen 16x, Ex 17x, Lev 13x, Num 7x, Dtn 30x; vgl, P.R. Davies/G.J. Brooke/P.R. Callaway, The Complete World of the Dead Sea Scrolls, 2002, 165.

44 Numeri (s. Anm. 1), 12. 
haben sollte ${ }^{45}$. Für Levin gehören nur 11,2a.4b*.11.23.31-32* zu J, die restlichen Verse sind Fortschreibungen ${ }^{46}$. Van Seters betrachtet hingegen das gesamte Kapitel als eine von J geschaffene Komposition ${ }^{47}$. Ähnliche Unsicherheiten bestehen bzgl. Num 12. Seebass will die nach ihm usprüngliche Erzählung über Miriams Kritik an Moses ausländischer Frau $J$ zuschreiben, selbst wenn »es keine sicheren Hinweise der Zugehörigkeit« gibt ${ }^{48}$; anders Levin: ganz Num 12 sei als JE und damit als nachjahwistisch zu verstehen ${ }^{49}$; Van Seters sieht auch dieses Kapitel als einheitlich $\mathrm{J}$ zugehörend an ${ }^{50}$. Das Fehlen eines Konsenses offenbart zunächst, dass die zitierten Autoren mit dem Siglum J höchst unterschiedliche Vorstellungen verbinden. Weiterhin stellt sich die Frage, ob die Zuschreibung des Grundbestandes oder der Ganzheit von Num 11-12 an J (oder $\mathrm{D}^{51}$ ) der Spezifizität dieser Texte wirklich Rechnung trägt. M.E. ist in der Aufeinanderfolge von Num 11-12 eine mehrstufige redaktionelle Schichtung erkennbar, die sich am besten mit dem Modell der Fortschreibung erklärt.

\subsection{Fortschreibung in Num 11-1252}

Die Existenz zweier Themen (Wachteln, Geistbegabung der 70 Ältesten) in Num 11 steht ausser Zweifel. Allerdings ist es schwierig aus Num 11 zwei vollständige voneinander unabhängige Geschichten zu rekonstruieren, denn das Leitwort תס findet sich innerhalb der beiden Erzählstränge (V. 4.16.22.24.30.32 [2x $]^{53}$ ). Ausgangspunkt der Textentwicklung ist eine im Gegensatz zu Ex 16 ins Negative gewendete Speiseerzählung ${ }^{54}$, die den Rebellionszyklus in Num 11-20* er-

45 Seebass, Numeri (s. Anm. 33), 31-41, dort auch eine gute Darstellung früherer Lösungsvorschläge. Vgl. auch P.J. Budd, Numbers, WBC 5, 1984, 124-127.

46 Levin, Jahwist (s. Anm. 17), 370-375.

47 Van Seters, Life (s. Anm. 15), 226-234.

48 Numeri (s. Anm. 33), 67, die Einschaltung der v. 2-9* schreibt er dem Jehowisten zu, vgl. 65.

49 Jahwist (s. Anm. 17), 375.

50 Life (s. Anm. 15), 234-239

51 So z.B. Blum, Studien (s. Anm. 19), 76-85, der Num 11-12 der «D-Komposition» zuschreibt, welche allerdings nicht näher bestimmte ältere Traditionen integriert. Vgl. ähnlich E. Aurelius, Fürbitter (s. Anm. 8), 178.

52 Vgl. zum folgenden auch T. Römer, Nombres 11-12 et la question d'une rédaction deutéronomique dans le Pentateuque, in: Deuteronomy (s. Anm. 25), 481-498.

53 V. 4.22 .32 gehören zur Wachtelerzählung, V. 16.24 und $30 \mathrm{zu}$ der Geistausgiessung.

54 Auch im Rahmen der Quellentheorie wurde die Wachtelerzählung generell als älter angesehen, vgl, aber Seebass, Numeri (s. Anm. 33), 36-40. 
öffnet. Möglicherweise setzt Num 11* bereits die Kombination von Manna und Wachteln in Ex 16 voraus und schreibt diesen Text midraschartig fort, um auf die ständige Rebellion des Volkes abzuheben. Dieser bereits nachexilische Text wurde dann anhand der Themen »Geistbegabung « und »Entlastung des Mose« von einem Redaktor überarbeitet, welcher Num 11 nun zu einer Gegenüberstellung von »Fleisch « und »Geist《 umgestaltet ${ }^{55}$. Die hier tätige Redaktion kann als »prophetisch-charismatisch « bezeichnet werden, denn es bestehen auffällige Parallelen zu der nachexilischen Heilsprophetie. Die Idee, dass ein Teil von Moses Geist auf die Ältesten übertragen wird, ist weder »jahwistisch « noch »dtr«; sie ist eigentlich nur mit Jes 63,11 vergleichbar ${ }^{56}$. Weiterhin bestehen enge Parallelen zu Ez (vgl. V. 4 mit Ez 37,11; V. 17 mit Ez 37,5.14; V. 29 mit Ez 36,27; 39,29 und auch Joel 3,1) und Deutero-bzw. Trito-Jesaja (vgl. V. 23 mit Jes 50,2; $59,1)^{57}$.

F. Crüsemann hat zu Recht bemerkt, dass prophetische Anliegen kaum Eingang in die Torah gefunden haben ${ }^{58}$. Anscheinend gab es nur im Numeribuch eine Möglichkeit die Hoffnung auf ein »allgemeines Prophetentum« unterzubringen. Die Gabe der Prophetie an das durch die 70 Ältesten repräsentierte Volk ${ }^{59}$ macht die dtr-mosaische Mediation im Grunde überflüssig (nach V. 30 zieht Mose sich in der Tat zurück). Die in Num 11 zum Ausdruck kommende prophetisch-charismatische Konzeption Israels blieb jedoch nicht unwidersprochen, sondern wurde bereits im folgenden Kapitel korrigiert ${ }^{60}$.

Von den beiden in Num 12 vorliegenden Streitthemen, die grosso modo auf V. 1,10-15 (Streit um Moses ausländische Frau) und V. 2-9 (Streit um Moses

55 Cf. H.-C. Schmitt, Die Suche nach der Identität des Jahweglaubens im nachexilischen Israel, in: J. Mehlhausen (Hg.), Pluralismus und Identität, 1995, 259-278, 276.

56 Num 27,18 und Dtn 34,9 erwähnen die Geisterfültheit Josuas. Diese nachpriesterlichen Texte wollen möglicherweise Num 11 korrigieren, welcher eine Demokratisierung des Geistes anstrebt. Nach Otto wären beide Texte einer Pentateuchredaktion, zuzuschreiben (Deuteronomium [s. Anm. 38], 226-228). Die Erwähnung Josuas zielt jedoch m.E. eher auf einen Hexateuch.

57 Die Evozierung mütterlicher Bilder in dem Streit zwischen Mose und Jhwh in V. 11-13 ist mit den auf Jhwh angewandten Mutterbildern in Jes 42, I4; 46,3; 49,15 zu vergleichen.

58 F. Crüsemann, Le Pentateuque, une Tora. Prolégomènes à l'interprétation de sa forme finale, in: A. de Pury (Hg.), Le Pentateuque en question, 1991², 339-360; 357: »cet ouvrage géant est remarquablement a-prophétique et an-eschatologique«.

59 Das Thema der 70 priviligierten Ältesten ist aus Ex 24,9-11 übernommen worden (vgl. das seltene אצ Ex 24,11 und Num 11,17.25). Dieser Text ist nach Ska nach-priesterlich und nach-dtr, vgl. J.L. Ska, Le repas d'Ex 24,11, Bib. 74 (1993), 305-327.

60 Diese Korrektion wird auch an dem textkritischen Problem von V. 25 deutlich: auf der Ebene der prophetischen Redaktion ist das gut bezeugte $¥ 10$ zu lesen: »sie hörten nicht auf«; auf der Ebene der Endredaktion hingegen: »sie fuhren nicht fort« ( $\left(0^{\circ}\right)$. 
Sonderstellung) aufzuteilen sind, können letztere Verse als direkte Antwort auf Num 11 verstanden werden ${ }^{61}$. L. Perlitt hat zu Recht festgestellt »Num. 11 wollte bestimmte Propheten mit Mose zusammenbringen; Num. 12 will die Trennung mit allen $\aleph^{62}$. Num 12,2ff. hebt in der Tat auf die Sonderstellung Moses $a^{63}$ : Nur Mose sieht die ${ }^{64}$, er ist Jhwhs Haushalter par excellence und kein anderer Prophet ist ihm vergleichbar. Dies ist eine klare Absage an die Auffassung von Num 11. Num 12,6-8 bereitet den Abschluss des Pentateuchs in Dtn 34,10-12 vor und ist wohl derselben Pentateuchredaktion zuzuschreiben ${ }^{65}$. Schliesslich ist diese Aufwertung des Mosebildes von einer späteren Redaktion benutzt worden, die mit V. 1 und 10-15 durch Mose die Mischehen legitimiert und somit der in Esr-Neh bezeugten exklusivistischen Tendenz kritisch gegenübersteht. Miriam wird wegen ihrer Kritik an der kuschitischen (schwarzen ?) Frau Moses durch eine Hautkrankeit »weiss wie Schnee « ${ }^{66}$ und kann allein durch die Interzession Moses geheilt werden ${ }^{67}$. Vielleicht spiegeln sich hier Anliegen eines Diasporajudentums ${ }^{68}$, wie sie im Pentateuch noch im Josephsroman zur Sprache kommen.

Somit können in Num 11-12 späterer Zusätze ungeachtet vier verschiedene Stadien der Textentstehung unterschieden werden. Die drei Redaktionen (Geistbegabung, Unvergleichbarkeit Moses, Legitimation der Mischehen) spielen eher eine marginale Rolle im Rahmen des Pentateuch und sind erst in einer sehr späten Phase eingefügt worden; deshalb konnten sie wohl nur noch im Buch Numeri Aufnahme finden. So wird man auch für den Anfang der nicht-priesterlichen Texte in Num auf die Annahme durchgehender Quellen zu verzichten haben.

61 Gegen Noth (Numeri [s. Anm. 1], 82-83) u.a., die V. 1 und 10-15* als Grundschicht (J) betrachten und V. 2-9 als spätere Erweiterung.

62 L. Perlitt, Mose als Prophet (1971) = Deuteronomium-Studien, FAT 8, 1994, 1-19, 6 .

63 Die Aussage über den unvergleichlichen Sanftmut Moses in v. 3 will das Bild eines eher gereizten Mose in Num 11 modifizieren. Oft wird V. 3 als Einschub angesehen, was aber nicht unbedingt nötig ist, so zu Recht Seebass, Numeri (s. Anm. 33), 69.

64 Diese Aussage kann als Präzision oder Korrektur zu Dtn 4,12.15 verstanden werden, welche feststellen, dass während der Theophanie am Gottesberg das Volk keine

65 Zur Funktion von Dtn 34,10-12 vgl. Römer/Brettler, Hexateuch (s. Anm. 38) und C. Frevel, Abschied (s. Anm. 3).

66 Zu einem möglichen Zusammenhang zwischen Miriams Kritik und ihrer Strafe vgl. J. Milgrom, Numbers, JPS Torah Commentary, 1990, 97.

67 Auch der Priester Aaron wird eindeutig Mose untergeordnet: er kann nur den Aussatz feststellen (vgl. V. 10 mit 2Chr 26,20), die Anweisungen zur Quarantäne kommen jedoch von Gott durch Mose.

68 Vgl. dazu B.J. Diebner, »... for he had married à Cushite woman« (Num 12,1), Nubica 1/II (1990), 499-504, der hier Anliegen der Juden aus Elephantine ausgedrückt sieht. 


\section{3. "Die Spuren ... verlieren sich" ${ }^{69}$. Der unauffindbare Jahwist in Numeri}

Die für Num 11-12 geführte Untersuchung müsste nun auch auf Num 13-25* ausgedehnt werden, was hier nicht zu bewerkstelligen ist. Für die nichtpriesterlichen Texte in Num 16-17, 20-21 und 25 mehren sich die Stimmen, die eine späte nicht-quellenhafte Entstehung dieser Texte postulieren ${ }^{70}$. Bleibt die Kundschaftergeschichte Num 13-14 für die jüngst noch L. Schmidt eine rekonstruierbare jahwistische Grundschicht postuliert hat ${ }^{71}$, um jedoch gleich zu konzedieren, dass deren Anfang »nicht erhalten $^{72}$ ist. Die Vielschichtigkeit von Num 13-14 steht außer Zweifel; zweifelhaft ist jedoch die Zugehörigkeit der nichtpriesterlichen Textteile zu einer jahwistischen Grundschicht. O. Artus unterscheidet einen »récit ancien« und einen »récit sacerdotal«, welche »ont été secondairement renrichis de plusieurs radditions postdeutéronomistes, dont la conséquence est de faire coexister dans le texte définitif de $\mathrm{Nb} 13-14$ deux théologies dont les perspectives diffèrent ${ }^{73}$. Der nichtpriesterliche Text könnte auf eine mündliche Einzeltradition über Kaleb zurückgehen. $\mathrm{Zu}$ einem vergleichbaren Ergebnis kommt E. Otto, welcher feststellt: $\gg$ Eine Urkundenhypothese bewährt sich in Num 13f. also nicht ${ }^{74}$. Nach Otto ist die nicht näher situierte Grunderzählung zunächst von einem Deuteronomisten in Dtn 1 eingebaut worden, danach wurde sie von der Hexateuchredaktion in Num 13-14 übernommen und ausgestaltet. In diesem Modell lässt sich aus der Grunderzählung keine den

69 Levin, Jahwist (s. Anm. 17), 50.

70 Zu Num 16-17 vgl. Levin, Jahwist, 377-378 : »nachendredaktionell«; Artus, Etudes (s. Anm. 33), 193-197: unabhängige Datan-Abiram Geschichte und verschiedene »relectures«; Ulrike Schorn, Rubeniten als exemplarische Aufrührer in Num. 16f*/Deut.11, in: Rethinking (s. Anm. 13), 251-268, 261: Datan-Abiram Geschichte als »nachträgliche theologische Umgestaltung und Endredaktion der schon vorhandenen priesterschriftlichen Passagen«. Num 20,14 21: S. Mittmann, Num 20,14-21 - eine redaktionelle Kompilation, in: H. Gese, (Hg.), Wort und Geschichte, FS K. Elliger, AOAT 18, 1973, 143-149; W. Oswald, Die Revision des Edombildes in Numeri xx 14-21, VT 50 (2000), 218-232: »postpriesterschriftlich«; Num 21,1-9: Levin, Jahwist, 379, »nachendredaktionell«; E. Aurelius, Fürbitter (s. Anm. 8), 147: "Kombination aus mehreren vorhergehenden Murrgeschichten «. Num 21,10-20 ist schon von $M$. Noth als spätes redaktionnelles Werk bezeichnet worden (Numeri [s. Anm. 1], 139), vgl. auch Levin, Jahwist, 379-380. Num 25: Knauf, Midian (s. Anm. 26), 160-165 : frühestens »nachjehovistisch«; B. Gosse, L'alliance avec Lévi et l'opposition entre les lignées royale et sacerdotale à l'époque perse, Trans 10 (1995), 29-33.

71 L. Schmidt, Die Kundschaftererzählung in Num 13-14 und Dtn 1,19-46, ZAW 114 (2002), 40-58, vgl. auch Seebass, Numeri (s. Anm. 33), 76ff.

72 Schmidt, ebda., 41.

73 O. Artus, Etudes (s. Anm. 33), 157.

74 E. Otto, Deuteronomium (s. Anm. 38), 58. 
Pentateuch durchlaufende Quelle eruieren. Auch der Ansatz von N. Rabe lässt den Jahwisten aus Num 13-14 verschwinden. Nach einer instruktiven Forschungsgeschichte, welche die Schwierigkeit aufzeigt, in Num 13-14 zwei bzw. drei unabhängige Quellen zu rekonstruieren, legt Rabe eine Analyse vor, nach welcher die Grundschrift aus den sog. priesterlichen Versen von Num 13-14 besteht und die nicht-priesterlichen Elemente als spätere Ergänzungen zu beurteilen $\operatorname{sind}^{75}$. Selbst wenn sich aus diesen Neuansätzen kein Konsens über die Entstehung von Num 13-14 erkennen lässt, so zeigt sich desungeachtet, dass auf die Hypothese eines Jahwisten zu verzichten ist. Damit stellt sich nun die Frage nach der Entstehung des Numeribuches und seiner Verbindung zum Tetrateuch.

\section{Das Ende des Jahwisten und die Entstehung von Numeri-einige abschlies- sende Bemerkungen}

Den obigen Überlegungen zufolge ist das Buch Numeri erst nachpriesterlich entstanden und zwar im Zusammenhang mit der Publikation eines Pentateuchs, in welchem sich die verschiedenen theologischen Strömungen des Judentums wiederfinden konnten. Insofern ist Otto recht zu geben, wenn er in Num hauptsächlich späte Penta- und Hexateuchredaktionen unterscheidet. Bleibt jedoch die Frage nach der Herkunft der Wüstentradition, die den Erzählungen in Num 1125 zugrunde liegt ${ }^{76}$. Ausserhalb des Pentateuchs ist eine solche erstmals bei Hosea belegt. Die Stellen, die in Hos die Wüstenzeit erwähnen $(2,5.16-17 ; 9,10$; $13,5)$, sind nach T. Dozemann jedoch nicht von den Erzählungen in Num abhängig, sondern stehen eher am Anfang der literarischen Fixation einer Wüstenzeittradition $^{77}$. In Hos (wie auch in Jer 2,2-9) ist die Zeit der Wüste weder geographisch näher bestimmt noch mit einem Itinerar verbunden, מדברָר bezeichnet vielmehr die Zeit der Fürsorge Jhwhs für Israel. In Hos 9,10 stellt die Erwähnung von Baal-Peor den Wendepunkt im harmonischen Verhältnis zwischen Israel und seinem Gott dar. Im Gegensatz zu Num markiert in Hos 9,10 das Hei-

75 N. Rabe, Vom Gerücht zum Gericht. Die Kundschaftererzählung Numeri 13.14 als Neuansatz in der Pentateuchforschung, THLI 8, 1994.

76 Die Bileamerzählungen brauchen hier nicht berücksichtigt werden, da diese sich ohne weiteres als eigenständige Tradition erkennen lassen.

77 T.B. Dozeman, Hosea and the Wilderness Wandering Tradition, in: Rethinking (s. Anm. 13), 55-70. 
ligtum von Baal-Peor den Eintritt in das Kulturland ${ }^{78}$. Die hoseanische Kritik des anscheinend populären israelitisch-moabitischen Grenzheiligtums kann als Vorlage für Num 25 verstanden werden ${ }^{79}$. Wenn Hosea den literarischen Anfang der Wüstentradition darstellt, so ist diese über Ex 16-18 in Numeri negativ rezipiert worden ${ }^{80}$. Es ist oft aufgefallen, dass im Unterschied zu Ex die Numeritexte ein weitaus konfliktreicheres Bild von Israels Wüstenaufenthalt zeichnen ${ }^{81}$. Dies erklärt sich dadurch, dass in Num die Wüste zum Schauplatz von politischen und ideologischen Machtkämpfen wird, welche die komplexe Situation der judäischen Gesellschaft im 5. Jh. vor der Promulgation der Torah wiederspiegelt.

Wenn man Num 11-25 mit Dtn 1-3 vergleicht ${ }^{82}$, so fällt auf, dass die Anfangskapitel des Dtn neben der Kundschaftererzählung hauptsächlich ${ }^{83}$ von der Eroberung des Ostjordanlandes berichten (Dtn 2--3, vgl. Num 20,10-21; 21,10$35 ; 32$ ), zu welcher die Aussendung von Spionen eine gute Einleitung darstellt. Anscheinend gab es eine kriegerische Tradition der Besetzung des Ostjordanlandes, welche in Dtn 1-3 zum erstenmal verschriftet wurde und dann in Numeri aufgenommen und ausgebaut wurde ${ }^{84}$. Wenn man vor Spekulationen nicht zurückschreckt, könnte man erwägen, ob nicht zunächst das Dtn, nach seiner Abtrennung, vom DtrG direkt an Lev 27 angefügt wurde. Die Wüstentradition und Dtn 1-3 hätte dann dtr, priesterlichen und anderen Kreisen den Ausgangspunkt geliefert durch die allmähliche Komposition von Numeri den Übergang etwas weniger abrupt zu gestalten. Das Buch Numeri wurde dadurch zum Schauplatz schriftgelehrter Interpretationen und Diskussionen, wie sie sich später in Mischnah und Talmud wiederfinden.

78 So E. Bons, Das Buch Hosea, NSK.AT 23/1,1996, 120-121 und G. Boudreau, Hosea and the Pentateuchal Traditions, in: M.P. Graham u.a. (Hg.), History and Interpretation, FS J.H. Hayes, JSOT.S 173, 1993, 121-132, 126.

79 Zur späteren Entstehung von Num 25 sowie zu Dtn 4,3 und Ps 106,28-31 vgl. G. Boudreau, ebda.

80 Ausserhalb des Pentateuch sind von den Wüstenerzählungen in Num nur Datan und Abiram (Ps 106,17) und (die Wasser von) Meriba belegt (Ps 81,8; 95,8; 106,32 und als geographische Angabe in Ez 47,19 und 48,28).

81 Vgl. z.B. T.L. Burden, The Kerygma of the Wilderness Traditions in the Hebrew Bible, American University Studies VII/163, 1994, 82-83.

82 Z.B. anhand der Tabelle bei J. Van Seters, The Pentateuch. A Social Science Commentary, $1999,149$.

83 Dtn 1,19-46 ist weit enger mit Ex 18 als mit Num 11 verwandt, da die Frage der Prophetie in Dtn 1 (noch) keinerlei Rolle spielt.

84 Dies würde die Positionen von Rose, Deuteronomist (s. Anm. 18); Van Seters, Pentateuch (s. Anm. 82), Aurelius, Fürbitter (s. Anm. 8) u.a. bestätigen, die Dtn 1-3 als Vorlage für die Erzählungen im Tetrateuch ansehen. 
Wie gesagt, dies ist recht spekulativ, und das Problem der Entstehung von Numeri ist damit nicht gelöst. Ein Jahwist darf jedoch dafür nicht mehr bemüht werden. Die Pentateuchdiskussion der letzten Jahre hat die Spezifika der »grösseren Einheiten« (Rendtorff) bzw. der einzelnen Bücher des Pentateuch wieder entdeckt. Von daher sollte überlegt werden, ob der Pentateuch für seine verschiedenen Komponenten nicht auch verschiedene redaktionsgeschichtliche Modelle erfordert ${ }^{85}$. Der Befund in Numeri spricht jedenfalls dafür.

\section{Abstract}

The most recent debate on the Pentateuch has made it probable that the conclusion of $\mathrm{P}^{g}$ can be found in Ex 40 or Lev 9; this means that $P$ can no longer be seen as the artist behind the Pentateuch. The Yahwist also cannot fill this role, as can be seen clearly in the book of Numbers. The non-priestly texts in Numbers are best explained by the principle of expansion. It seems that Numbers should be understood as a connection, composed in Persian times, between the priestly edition of the Tetrateuch and the book of Deuteronomy, which was separated from the deuteronomistic History.

85 Vgl. auch die Überlegungen bei Gertz, Tradition (s. Anm. 21), 388. 